
\title{
R Research Souture \\ Reasons for no good embryos in the IVF/ICSI cycle: A retrospective analysis.
}

\section{Kun Chu}

Changzheng Hospital

\section{Jing Shu}

Zhejiang Provincial People's Hospital

\section{Ling Zhang}

Zhejiang Provincial People's Hospital

\section{Wen Li}

Changzheng Hospital

Weihai Xu ( $\nabla$ xwh8023@sina.com)

Zhejiang Provincial People's Hospital https://orcid.org/0000-0001-5238-6521

\section{Research article}

Keywords: Good quality embryo, In-vitro fertilization, Intracytoplasmic sperm injection, Gamete

Posted Date: September 11th, 2019

DOI: https://doi.org/10.21203/rs.2.14279/v1

License: (c) (1) This work is licensed under a Creative Commons Attribution 4.0 International License. Read Full License 


\section{Abstract}

Background: The aim of this study was to clarify related factors of no good quality embryos in IVF/ICSI cycle so as to improve the IVF/ICSI outcome.

Methods: Data of Day 3 embryos and patients of 2518 cycles from January 2012 to December 2016 were analyzed in this study. A binary logistic regression model was conducted to examine the factors which led to no good quality embryo in one cycle.

Results: The incidence of no good quality embryo in one cycle was $13.3 \%$. Number of oocytes, fertilization methods, stimulation protocol, and fertilization rate were significant in logistic regression analysis. Further analysis showed significant correlations between increasing intracellular fragmentation and the following parameters: number of oocytes, fertilization rate, and infertility diagnosis; slow growing embryos: number of oocytes, fertilization rate, and fertilization methods; embryos with asymmetric cleavage: number of oocytes, fertilization rate, fertilization methods, female age, and male age; compound causes: number of oocytes, fertilization rate, infertility duration, stimulation protocol, and fertilization methods.

Conclusions: The results suggested that gamete poor quality is the main reason for the embryo quality decline, which underlines the importance to improve gamete quality and eventually the fertility outcome in clinical practice.

\section{Introduction}

The outcome of in-vitro fertilization/intracytoplasmic sperm injection-embryo transfer (IVF/ICSI-ET) is affected by various factors, of which embryo quality is one of the most important prognostic factors for pregnancy chance[1]. Good quality embryos have higher implantation potential, thus, resulting in higher pregnancy chance. Some patients do not have good quality embryos in the treatment cycle, thus resulting a lower pregnancy rate.

Integrated morphological cleavage embryo scoring is the most commonly used method to evaluate embryo quality of the cleavage stage. The number of blastomeres, the degree of fragmentation and the symmetry of the blastomeres are the main parameters[2]. Non-good quality embryos, such as slow growing embryo, embryos with asymmetric cleavage, and embryos with much intracellular fragmentation, can lead to a decline in embryo developing potential ${ }^{2}$. In most cases, good quality embryos and non-good quality embryos coexist in the treatment procedure. While in some rare cases, all embryos are non-good quality embryos, reducing the pregnancy chance, and its causes still lack of systematic analysis. It is of great significance to clarify its related factors, take relevant measures so as to reduce the incidence of non-good quality embryos and improve the fertility outcome of IVF/ICSI. This study was conducted to investigate the cause of no good quality embryos in one cycle by analyzing the related data of Day 3 embryo and patients undergoing IVF/ICSI treatment. 


\section{Materials And Methods \\ Study population}

The medical records of 4841 cycles from January 2012 to December 2016 at Reproductive Medicine Center of Changzheng Hospital and Zhejiang Provincial People's Hospital were retrospective analyzed. Exclusion criteria included unexplained infertility, infertility caused by more than one factor, oocyte/sperm reception, and either one of the couple with chromosomal abnormalities. A total of 2518 cycles with controlled ovarian hyperstimulation were included. The Ethics Committee of Changzheng Hospital and Zhejiang Provincial People's Hospital gave a positive approval for this retrospective study. Informed consent was obtained from all individual participants included in the study.

\section{Stimulation protocols}

The individual protocols were chosen by reproductive endocrionologists on a case-by-case basis. Ovulation was triggered when at least one leading follicle reached $18 \mathrm{~mm}$ or 2 reached $17 \mathrm{~mm}$, and oocytes retrieved was done by transvaginal ultrasound guided oocyte retrieval $34-36 \mathrm{~h}$ thereafter. Approximately 4 to 6 hours after follicular aspiration, standard fertilization in vitro or intracytoplasmic sperm injection was performed as appropriate. On Day 3, all the embryos were reassessed, and were then transferred or cryopreserved.

\section{Outcome measures}

Day-3 embryos comprising at least 6 cells, with less than 25\% intracellular fragmentation were defined as good quality. Cycles with at least one good quality embryo were included in the G-group, while others were included in the $\mathrm{N}$-group. Cycles in the $\mathrm{N}$-group were further divided into subgroups according to their characteristics: S-group, slow growing embryos with no more than 5 cells; A-group, embryos with asymmetric cleavage; F-group, embryos with no less than $25 \%$ intracellular fragmentation; C-group, compound causes. The clinical pregnancy was defined as visualization of at least one gestational sac and presence of a fetal heartbeat in approximately the 5th week after fertilization. Cumulative pregnancy rate and cumulative live birth rate were calculated after a complete IVF/ICSI cycle, which was defined as all transfer cycles in an IVF/ICSI cycle started with ovarian stimulation, ended with live birth or no available embryos or abandonment. Statistical analysis

Statistical analyses were carried out using the IBM SPSS Statistics Version 19.0. For quantitative variables, means and SDs were calculated, while frequencies and percentages were calculated in case of qualitative ones. Differences between quantitative variables were assessed by $t$ tests, and qualitative ones by Chi-square test. A binary logistic regression model was conducted to examine the factors which led to no good quality embryo in one cycle. Two-tailed $P$ value $<0.05$ was considered statistically significant. 


\section{Results}

Among the 2518 cycles included in this study, 336 cycles (N-group, 13.3\%) had no good quality embryo (S-group: 81; F-group: 81; A-group: 92; C-group: 82). There was no significant difference between G-group and $\mathrm{N}$-group in the aspect of age and rate of primary infertility $(P>0.05)$; while there was significant difference in the aspect of infertility diagnosis $(P<0.05)$, stimulation protocol $(P<0.01)$, infertility duration $(P<0.01)$, fertilization methods $(P<0.001)$, number of oocytes retrieved $(P<0.001)$, and fertilization rate $(P<0.001)$ (Table 1$)$. With regard to clinical outcomes, T-group had significant higher cumulative pregnancy rate $(81.8 \% \& 37.5 \%, \mathrm{P}<0.001)$ and cumulative live birth rate $(74.4 \% \& 30.7 \%, \mathrm{P}<0.001)$ than $\mathrm{N}$ group.

In logistic regression analysis for associations with no good quality embryo in one cycle, number of oocytes, fertilization methods, stimulation protocol, and fertilization rate remained significant. Further analysis showed significant correlations between increasing intracellular fragmentation and the following parameters: number of oocytes, fertilization rate, and infertility diagnosis; slow growing embryos: number of oocytes, fertilization rate, and fertilization methods; embryos with asymmetric cleavage: number of oocytes, fertilization rate, fertilization methods, female age, and male age; compound causes: number of oocytes, fertilization rate, infertility duration, stimulation protocol, and fertilization methods (Table 2).

According to the number of oocytes, cycles were divided into 1-5, 6-10,11-20 and $>20$ groups, of which the incidence of no good quality embryo was $28.1 \%$ (81/288), 17.9\% (141/787), 9.8\% (106/1087), and $2.2 \%(8 / 356)$ respectively. The incidence of no good quality embryo was negative correlation with number of oocytes, with significant differences between groups $(P<0.001)$.

When taking stimulation protocol into consideration, the incidence of no good quality embryo of short protocol, long protocol, and GnRH antagonist protocol was 11.4\% (189/1662), 19.0\% (136/717),and 6.6\% $(8 / 121)$ respectively. The incidence of no good quality embryo was significant higher in short protocol $(\mathrm{P}$ $<0.001)$.

The occurrence of no good quality embryo was significantly lower in the IVF group than that in the ICSI group $(10.1 \% \& 19.2 \%, P<0.001)$. When further considering the sperm retrieval method of ICSI, the incidence of no good quality embryo was significantly lower in the group of sperm by testicular sperm extraction(TESE) than that of sperm by percutaneous epididymal sperm aspiration(PESA) and sperm by ejaculation $(P<0.001)$, while there was no significant difference between the latter two groups $(P>$ 0.05) (Table 3.)

When analyzed based on fertilization rate, the incidence of no good quality embryo of $\leq 30 \% \otimes 31-60 \%$, and $>60 \%$ group was $50.5 \%(47 / 93), 18.8 \%(117 / 624)$ and $9.6(172 / 1801)$ respectively, with significant differences among these groups $(P<0.001)$.

\section{Discussion}


To the best of our knowledge, this is the first study to report the incidence rate of no good quality embryo in one cycle and analyze its causes. The incidence of no good quality embryo in one cycle of IVF/ICSI-ET treatment was $13.3 \%$. Growth retardation, increased fragmentation, asymmetric cleavage, and compound factors were common reasons with similar incidence. The result of logistic regression analysis showed number of oocytes, fertilization methods, stimulation protocol, and fertilization rate remained were all correlated with no good quality embryo in one treatment cycle.

Number of oocytes retrieved reflect the function of ovary, with aging being the main factor of the decline of the ovarian function[3]. Increasing maternal age leads to reduced mitochondrial DNA (mtDNA) levels, resulting in decrease of ATP production and increase of embryo fragmentation[4,5]. Advanced maternal age also results in an increase of oocyte chromosomal abnormalities, which leads to a decline in the oocyte quality and dysontogenesis[6]. Diseases, such as moderate to severe endometriosis, decrease the ovarian reserve function, affect the oocyte quality and embryo development[7]. Several studies have demonstrated, short protocol may result in fewer number of oocytes retrieved and fewer good quality embryos[8,9], which may be a reason why rate of no good quality embryos was highest in the short protocol group.

Fertilization method, decided by sperm and oocyte natural fertilization ability, is related to embryo development. For those whose sperm quality and natural fertilization ability are good, conventional IVF fertilization is chosen; while ICSI is chosen for those with oligoasthenozoospermia or with teratozoospermia, whose sperm quality and natural fertilization ability are poor. Our results showed lower dysontogenesis rate in the group of sperm by TESE, and this may be explained by sperm quality and its developing environment. The semen ROS level in those with oligoasthenozoospermia or with teratozoospermia is increased, with sperm DNA abnormalities increased, which may affected the development of embryo[10,11]. Obstructive azoospermia is often related to inflammation, affecting the environment of deferent duct, and long-term obstruction will decrease the sperm quality by increasing the sperm aging and death. Studies have shown that the quality of testicular sperm is better than that of epididymal sperm[12]. These may all be the reasons why no good quality embryo rate is lowest in the group of TESE.

\section{Conclusions}

We have identified, for the first time, the incidence rate of no good quality embryo in one cycle and its causes. Poor quality of sperm and oocyte in the treatment process is the main reason for the decline of embryo quality, and further reduce the treatment efficiency. For those with no good quality embryos in the treatment process, it is important to figure out how to improve the gamete quality, embryo quality, and eventually the fertility outcome.

\section{Abbreviations}


IVF/ICSI -ET: in-vitro fertilization/intracytoplasmic sperm injection - embryo transfer; TESE: testicular sperm extraction; PESA: epididymal sperm aspiration; mtDNA: mitochondrial DNA.

\section{Declarations}

Ethics approval and consent to participate: All procedures performed in studies involving human participants were in accordance with the ethical standards of the institutional and/or national research committee and with the 1964 Helsinki declaration and its later amendments or comparable ethical standards. Informed consent: Informed consent was obtained from all individual participants included in the study.

Consent for publication: All authors agree the publication of the manuscript.

Availability of data and material: Anonymous original data is available when necessary.

Competing interests: The authors declare that they have no competing interests.

Funding: None.

Authors' contributions: WX: project development, data collection, manuscript writing. WL: project development, data collection, manuscript writing. KC: data collection, manuscript writing. JS: methodology, data collection. LZ: methodology, data collection.

Acknowledgements: We thank the patients for participating in this study.

\section{References}

1.Niinimaki M, Veleva Z, Martikainen $\mathrm{H}$. Embryo quality is the main factor affecting cumulative live birth rate after elective single embryo transfer in fresh stimulation cycles. Eur J Obstet Gynecol Reprod Biol. 2015;194:131-135.

2. Holte J, Berglund L, Milton K, et al. Construction of an evidence-based integrated morphology cleavage embryo score for implantation potential of embryos scored and transferred on day 2 after oocyte retrieval. Hum Reprod. 2007;22(2):548-557.

3.Female age-related fertility decline. Committee Opinion No. 589. Fertil Steril. 2014;101(3):633-634.

4.Santos TA, El Shourbagy S, St John JC. Mitochondrial content reflects oocyte variability and fertilization outcome. Fertil Steril. 2006;85(3):584-591.

5.Fujimoto VY, Browne RW, Bloom MS, Sakkas D, Alikani M. Pathogenesis, developmental consequences, and clinical correlations of human embryo fragmentation. Fertil Steril. 2011;95(4):1197-1204. 
6. Wang ZB, Schatten H, Sun QY. Why is chromosome segregation error in oocytes increased with maternal aging? Physiology (Bethesda). 2011;26(5):314-325.

7.Shebl O, Sifferlinger I. Oocyte competence in in vitro fertilization and intracytoplasmic sperm injection patients suffering from endometriosis and its possible association with subsequent treatment outcome: a matched case-control study. 2017;96(6):736-744.

8.Ou J, Xing W, Li T, Li Y, Xu Y, Zhou C. Short versus long gonadotropin-releasing hormone analogue suppression protocols in advanced age women undergoing IVF/ICSI. Gynecol Endocrinol. 2016;32(8):622-624.

9.Ou J, Xing W, Li Y, Xu Y, Zhou C. Short versus Long Gonadotropin-Releasing Hormone Analogue Suppression Protocols in IVF/ICSI Cycles in Patients of Various Age Ranges. PLoS One. 2015;10(7):e0133887.

10.Agarwal A, Mulgund A, Sharma R, Sabanegh E. Mechanisms of oligozoospermia: an oxidative stress perspective. Syst Biol Reprod Med. 2014;60(4):206-216.

11.Varshini J, Srinag BS, Kalthur G, et al. Poor sperm quality and advancing age are associated with increased sperm DNA damage in infertile men. Andrologia. 2012;44 Suppl 1:642-649.

12.Hammoud I, Bailly M, Bergere M, et al. Testicular Spermatozoa Are of Better Quality Than Epididymal Spermatozoa in Patients With Obstructive Azoospermia. Urology. 2017;103:106-111.

\section{Tables}

\section{Table 1. Basal characteristics}




\begin{tabular}{|c|c|c|}
\hline Variables & $\begin{array}{l}\text { Group G } \\
\mathrm{N}=2182\end{array}$ & $\begin{array}{c}\text { Group } N \\
N=336\end{array}$ \\
\hline Mean age of maternal (y) & $30.9 \pm 4.1$ & $31.6 \pm 4.1$ \\
\hline Mean age of paternal (y) & $32.9 \pm 4.2$ & $33.4 \pm 4.1$ \\
\hline \multicolumn{3}{|l|}{ Type of infertility } \\
\hline Primary & $799(36.6 \%)$ & $120(35.7 \%)$ \\
\hline Secondary & $1383(63.4 \%)$ & $216(64.3 \%)$ \\
\hline \multicolumn{3}{|l|}{ Cause of infertility ${ }^{a}$} \\
\hline Tubal factor & $1419(65.0 \%)$ & 189(56.3\%) \\
\hline anovulatory & $75(3.4 \%)$ & $9(2.7 \%)$ \\
\hline Endometriosis & $61(2.8 \%)$ & $20(6.0 \%)$ \\
\hline Poor ovrian response & $16(0.7 \%)$ & $4(1.2 \%)$ \\
\hline Oligo-astheno-teratospermia & $448(20.5 \%)$ & $92(27.4 \%)$ \\
\hline Obstructive azoospermia & $123(5.6 \%)$ & $22(6.5 \%)$ \\
\hline Duration of infertility $(y)^{b}$ & $3.9 \pm 2.9$ & $4.1 \pm 3.3$ \\
\hline \multicolumn{3}{|l|}{ Protocol of $\mathrm{COS}^{\mathrm{a}}$} \\
\hline Long protocol & 1473(67.5\%) & 189(56.2\%) \\
\hline Short protocol & $581(26.6 \%)$ & $136(40.5 \%)$ \\
\hline GnRH antagonist protocol & $113(5.2 \%)$ & $8(2.4 \%)$ \\
\hline Others & $15(0.7 \%)$ & $3(0.9 \%)$ \\
\hline Number of oocytes ${ }^{a}$ & $13.5 \pm 7.0$ & $9.5 \pm 5.2$ \\
\hline \multicolumn{3}{|l|}{ Fertilization methods ${ }^{a}$} \\
\hline IVF & $1449(66.4 \%)$ & $162(48.2 \%)$ \\
\hline ICSI & 733(33.6\%) & $174(51.8 \%)$ \\
\hline Rate of fertilization ${ }^{\mathrm{a}}$ & $72.3 \pm 18.0$ & $60.1 \pm 24.4$ \\
\hline
\end{tabular}

Note ${ }^{\mathrm{a}} \mathrm{Q} \mathrm{P}<0.001 \square^{\mathrm{b}}: \mathrm{P}<0.01$

Table 2. Logistic regression analysis for association 


\begin{tabular}{|c|c|c|c|c|c|}
\hline Variables & Group N & Group F & Group A & Group S & Group C \\
\hline \multirow{2}{*}{$\begin{array}{l}\text { Number of } \\
\text { oocytes }\end{array}$} & $0.872 \llbracket 0.848-$ & $0.882 \square 0.839-$ & $0.890 \llbracket 0.847-$ & 0.935ロ0.892- & $0.842 \square 0.793-$ \\
\hline & $0.897 \square^{\mathrm{a}}$ & $0.928 \square^{\mathrm{a}}$ & $0.935 \square^{\mathrm{a}}$ & $0.980 \square^{\mathrm{b}}$ & $0.893 \square^{\mathrm{a}}$ \\
\hline \multirow{2}{*}{$\begin{array}{l}\text { Fertilization } \\
\text { rate }\end{array}$} & $0.969 \llbracket 0.963-$ & 0.987ロ0.977- & 0.977ロ0.968- & 0.963ロ0.954- & 0.973ロ0.963- \\
\hline & $0.975 \square^{\mathrm{a}}$ & $0.998 \square^{b}$ & $0.987 \square^{\mathrm{a}}$ & $0.973 \square^{\mathrm{a}}$ & $0.982 \square^{\mathrm{a}}$ \\
\hline \multirow{2}{*}{$\begin{array}{l}\text { Cause of } \\
\text { infertility }\end{array}$} & 1.011๘0.987- & 1.053ロ1.011- & 1.003ロ0.962- & $1.021 \square 0.980-$ & 0.947ロ0.892- \\
\hline & $1.036 \square$ & $1.096 \square^{\mathrm{b}}$ & $1.047 \square$ & $1.064 \square$ & $1.005 \square$ \\
\hline \multirow{2}{*}{$\begin{array}{l}\text { Type of } \\
\text { infertility }\end{array}$} & $0.808 \square 0.614-$ & 0.887ロ0.538- & 0.993ロ0.622- & $0.671 \square 0.402-$ & 0.837ロ0.499- \\
\hline & $1.065 \square$ & $1.461 \square$ & $1.585 \square$ & $1.119 \square$ & $1.403 \square$ \\
\hline \multirow{2}{*}{$\begin{array}{l}\text { Mean age of } \\
\text { maternal }\end{array}$} & 1.092凸0.973- & $0.962 \square 0.784-$ & $1.256 \square 1.068-$ & $1.022 \square 0.820-$ & $1.027 \square 0.822-$ \\
\hline & $1.225 \square$ & $1.179 \square$ & $1.476 \square^{\mathrm{b}}$ & $1.274 \square$ & $1.283 \square$ \\
\hline \multirow{2}{*}{$\begin{array}{l}\text { Mean age of } \\
\text { paternal }\end{array}$} & $0.899 \square 0.802-$ & 0.965ロ0.789- & $0.784 \llbracket 0.666-$ & 0.998ロ0.803- & $0.968 \square 0.776-$ \\
\hline & $1.007 \square$ & $1.179 \square$ & $0.922 \square^{\mathrm{b}}$ & $1.241 \square$ & $1.207 \square$ \\
\hline \multirow{2}{*}{$\begin{array}{l}\text { Duration of } \\
\text { infertility }\end{array}$} & 0.998ロ0.956- & $1.041 \square 0.961$ - & $1.001 \square 0.932-$ & 1.033ロ0.961- & 0.915ロ0.840- \\
\hline & $1.041 \square$ & $1.128 \square$ & $1.076 \square$ & $1.109 \square$ & $0.996 \square^{\mathrm{C}}$ \\
\hline \multirow[t]{2}{*}{ Protocol of COS } & $0.854 \llbracket 0.748-$ & $0.846 \sqcap 0.654-$ & $1.009 \llbracket 0.826-$ & $0.861 \square 0.662-$ & $0.757 \llbracket 0.578-$ \\
\hline & $0.974 \square^{\mathrm{C}}$ & $1.093 \square$ & $1.231 \square$ & $1.120 \square$ & $0.9911^{\mathrm{C}}$ \\
\hline \multirow{2}{*}{$\begin{array}{l}\text { Rate of } \\
\text { fertilization }\end{array}$} & $1.788 \square 1.345-$ & 0.866ロ0.503- & 1.688ロ1.031- & $2.267 \square 1.341-$ & $2.021 \square 1.214-$ \\
\hline & $2.376 \rrbracket^{\mathrm{a}}$ & & $2.762 \square^{\mathrm{C}}$ & $3.834 \square^{b}$ & $3.363 \rrbracket^{b}$ \\
\hline
\end{tabular}

s with no good quality embryos in IVF/ICSI cycle.

Note: ${ }^{\mathrm{a}}: P<0.001 ;{ }^{\mathrm{b}}: P<0.01{ }^{\mathrm{c}}: P<0.05$

\section{Table 3. Different sperm retrieval methods of ICSI.}

\begin{tabular}{lccc}
\hline Variables & Cycles & Group G & Group N (Rate) \\
\hline ICSI-E & 756 & 604 & $152(20.1 \%)$ \\
\hline ICSI-P & 43 & 34 & $9(20.9 \%)$ \\
ICSI-T & 108 & 95 & $13(12.0 \%)$ \\
\hline
\end{tabular}

Note: ICSI-E: sperm by ejaculation; ICSI-P: sperm by percutaneous epididymal sperm aspiration(PESA); ICSI-T: sperm by testicular sperm ectraction(TESE) 